\title{
Construction of discrete-time models for port-controlled Hamiltonian systems with applications
}

\author{
Dina Shona Laila and Alessandro Astolfi * \\ Electrical and Electronic Engineering Department \\ Imperial College London \\ Exhibition Road, London SW7 2AZ, UK \\ E-mail: $\{$ d.laila, a.astolfi\}@imperial.ac.uk
}

8th May 2005

\begin{abstract}
The issues of constructing a discrete-time model for Hamiltonian systems are in general different from those for dissipative systems. We propose an algorithm for constructing an approximate discrete-time model, which guarantees Hamiltonian conservation. We show that the algorithm also preserves, in a weaker sense, the losslessness property of a class of port-controlled Hamiltonian systems. An application of the algorithm to port-controlled Hamiltonian systems with quadratic Hamiltonian is presented, and we use this to solve the stabilization problem for this class of systems based on the approximate discrete-time model constructed using the proposed algorithm. We illustrate the usefulness of the algorithm in designing a discrete-time controller to stabilize the angular velocity of the dynamics of a rigid body.
\end{abstract}

Keywords: Hamiltonian systems; Discrete-time systems; Hamiltonian conservation; Stabilization; Nonlinear systems.

\section{Introduction}

One of the main issues in direct discrete-time design for a nonlinear sampled-data control system is the costruction of a good model to use for the design. Even if the continuous-time model of a nonlinear plant is known, we cannot in general compute the exact discrete-time model of the plant, since it requires computing an explicit analytic solution of a nonlinear differential equation. Therefore, to solve this problem, an approximate model of the plant is used instead.

${ }^{*}$ This research was supported by the EPSRC Portfolio Award, Grant No. GR/S61256/01. The work of A. Astolfi is partly supported by the Leverhulme Trust. 
A general framework for stabilization of sampled-data nonlinear systems via their approximate discrete-time model was presented in $[4,5]$. In these works the problems are seen from the framework of dissipative systems and hence approximate discrete-time models can be obtained using various numerical algorithms, such as Runge-Kutta and multistep methods. Consistency properties are then used to measure the discrepancies between the approximate and the exact models (see $[4,5,9])$. Unfortunately, most numerical methods that apply to dissipative systems do not apply to conservative systems, in the sense that those methods would destroy the conservative property of the systems. As a results, we may not be able to use these methods to obtain a good model for conservative systems, especially when control design exploits the conservative property of the plant.

Hamiltonian systems are an important class of nonlinear systems commonly used to model conservative physical systems, e.g. electrical networks, rigid manipulators, satellites, vehicles etc. There has been a lot of research to develop numerical algorithms that preserve important properties of Hamiltonian conservative systems, particularly conservation of the symplectic mapping and Hamiltonian conservation, which are two of the most important properties of these systems (see for example $[9,1]$ and references therein). However, to the best of the authors knowledge, there is only very few research in the direction of direct discrete-time controller design. Motivated by the importance of Hamiltonian systems in control theory and applications and the prevalent use of computers to control such systems, in this paper we study the stabilization problem for Hamiltonian conservative systems via approximate discrete-time models. We need to mention that although it is possible to model non-conservative systems using a Hamiltonian approach, we restrict the use of the term Hamiltonian systems only for Hamiltonian conservative systems.

In this paper we use some available results in the literature to propose a numerical recipe for constructing a discrete-time model for a class of Hamiltonian systems. We show that the algorithm guarantees the Hamiltonian conservation in the absence of control inputs. We present an application of the algorithm to port-controlled Hamiltonian systems, where the control is taken into account. We show that the algorithm also preserves a type of losslessness property of port-controlled Hamiltonian systems under sampling and we illustrate the usefulness of this result as a tool to solve a direct discrete-time stabilization design.

The paper is organized as follows. In Section 2, we present some preliminaries. Main results are presented in Section 3, followed by an application in Section 4. The stabilization problem is discussed in Section 5, and the paper is closed with a summary in Section 6 . 


\section{Preliminaries}

\subsection{General notions and notation}

The set of real and natural numbers (including 0) are denoted respectively by $\mathbb{R}$ and $\mathbb{N}$. We denote $x_{k}:=x(k T)$ and $x_{k+1}:=x((k+1) T), \forall k \in \mathbb{N}$. We also introduce the notation

$$
x_{\frac{1}{2}}=\frac{1}{2}\left(x_{k+1}+x_{k}\right) \text {. }
$$

For a matrix $J$, the matrices $J^{\top}$ and $J^{+}$denote the transpose and the Moore-Penrose inverse of $J$, respectively and the following hold:

$$
\begin{gathered}
J J^{+} J=J \text { and } J^{+} J J^{+}=J^{+} \\
\left(J^{+} J\right)^{\top}=J^{+} J \text { and }\left(J^{+} J\right)^{2}=J^{+} J .
\end{gathered}
$$

Moreover, if $J$ is skew symmetric, then the followings hold:

$$
\begin{aligned}
J^{\top} & =-J \\
x^{\top} J x & =0 .
\end{aligned}
$$

If $J$ is invertible with inverse $J^{-1}$, then $J^{+}=J^{-1}$.

\subsection{Port-controlled Hamiltonian systems}

In this paper we focus on Hamiltonian systems with collocated inputs and outputs, namely portcontrolled Hamiltonian systems [10]. A general representation of port-controlled Hamiltonian systems is as follows:

$$
\begin{aligned}
& \dot{x}=J(x) \nabla H(x)+g(x) u \\
& y=g^{\top}(x) \nabla H(x),
\end{aligned}
$$

where $x \in \mathbb{R}^{n}, u \in \mathbb{R}^{m}$ and $y \in \mathbb{R}^{m}$ are the state, input and output vectors, respectively. $\nabla H(x)$ is the gradient of the function $H$ with respect to the vector $x . J(x)$ is an $n \times n$ skew-symmetric matrix with entries depending smoothly on $x$. Note that $J(x)$ is not necessarily non-singular. System (6) is called a port-controlled Hamiltonian systems with the structure matrix $J(x)$ and Hamiltonian function $H(x)$. It can be seen that $\frac{d}{d t} H(x(t))=u^{\top}(t) y(t)$, showing that the system (6) is conservative if $H \geq 0$ (see Section 4.2 of [10]).

Another important property of port-controlled Hamiltonian systems which may be inferred from the structure of $J(x)$, is the existence of Casimir functions $C(x)$. These functions are independent of the Hamiltonian $H(x)$. If for a port-controlled Hamiltonian system there exists a function $C(x)$ which is a solution of

$$
(\nabla C(x))^{\top} J(x)=0 \quad \forall x,
$$


then along the trajectories of the system we have

$$
\frac{d C(x)}{d t}=(\nabla C(x))^{\top} g(x) u .
$$

It is obvious that for $u=0$ (or for arbitrary input if $(\nabla C(x))^{\top} g(x)=0$ ), then $\frac{d C(x)}{d t}=0$ and the function $C(x)$ is conserved. For more details about port-controlled Hamiltonian systems, we refer to [10].

Example 2.1 [10]. An important example of port-controlled Hamiltonian systems is a controlled rigid body spinning around its center of mass in the absence of gravity, which is commonly used to model a satellite. The energy variables are the three components of the angular momentum $p$ along the three principal axes $p=(\alpha, \beta, \gamma)^{\top}$, and the energy is the kinetic energy

$$
H(p)=\frac{1}{2}\left(I_{\alpha} \alpha^{2}+I_{\beta} \beta^{2}+I_{\gamma} \gamma^{2}\right),
$$

where $I_{\alpha}, I_{\beta}, I_{\gamma}$ are the principal moments of inertia. The Euler's equations for the rigid body subject to a uniformly weighted control torque are

$$
\left[\begin{array}{c}
I_{\alpha} \dot{\alpha} \\
I_{\beta} \dot{\beta} \\
I_{\gamma} \dot{\gamma}
\end{array}\right]=\left[\begin{array}{ccc}
0 & -\gamma & \beta \\
\gamma & 0 & -\alpha \\
-\beta & \alpha & 0
\end{array}\right]\left[\begin{array}{c}
\frac{\partial H}{\partial \alpha} \\
\frac{\partial H}{\partial \beta} \\
\frac{\partial H}{\partial \gamma}
\end{array}\right]+\left[\begin{array}{l}
1 \\
1 \\
1
\end{array}\right] u .
$$

Note that in general we can have non-uniform weight factors for the control input. The corresponding collocated output is given by

$$
y=\alpha+\beta+\gamma .
$$

It is obvious that the system is a port-controlled Hamiltonian system, and it is easy to see that the time derivative of the Hamiltonian is

$$
\frac{d H}{d t}=u^{T} y
$$

Hence, as $H \geq 0$, the system is conservative.

\subsection{Numerical methods for Hamiltonian conservation}

Most of the commonly known numerical methods are used for modeling the dynamics of dissipative systems. The issues of modeling Hamiltonian systems are in general quite different from that of dissipative systems. Numerical methods that define the dynamics of dissipative systems do not necessarily define the dynamics of Hamiltonian systems, in the sense that most of the methods would destroy Hamiltonian conservation.

In this subsection a review of a numerical method that can be used to model Hamiltonian conservative systems with no input is presented $[1,9]$. The method involves evaluation of the Hamiltonian $H(\cdot)$ and its gradient $\nabla H(\cdot)$, and it is given by:

$$
\begin{aligned}
\frac{x_{k+1}-x_{k}}{T}= & J\left(x_{\frac{1}{2}}\right) \nabla H\left(x_{\frac{1}{2}}\right) \\
& -J\left(x_{\frac{1}{2}}\right)\left[H\left(x_{k+1}\right)-H\left(x_{k}\right)+\left\langle\nabla H\left(x_{\frac{1}{2}}\right), x_{k+1}-x_{k}\right\rangle\right] \frac{x_{k+1}-x_{k}}{\left\|\left(x_{k+1}-x_{k}\right)\right\|^{2}},
\end{aligned}
$$


where $J$ is a skew symmetric matrix that satisfies not only (4), but

$$
J^{\top}=-J=J^{-1} .
$$

We refer to the algorithm (13) as Algorithm ALG1. The method is a second order accurate automatic conserving method that directly enforce Hamiltonian conservation and applies for systems of arbitrary even dimension. Note that the method is a perturbation of the implicit midpoint rule (see for instance $[8,9]$ and references therein). The perturbation is sufficiently small to retain second order accuracy. The convergence of this automatic Hamiltonian conserving method is guaranteed by Theorem 8.5.4 of [9], which we restate as Theorem 2.2 below.

Assumption 2.1 [9] The function $H \in C^{\infty}\left(\mathbb{R}^{2 N}, \mathbb{R}\right)$, with $N \in \mathbb{N}-\{0\}$ is such that for some $a \in \mathbb{R}$, the system (6) with $u=0$, defines a dynamical system on a compact set $B_{a}$, which is defined as

$$
B_{a}:=\left\{x \in \mathbb{R}^{2 N}: H(x)=a\right\},
$$

and it is such that

$$
\min _{x \in B_{a}}\|\nabla H(x)\|>0,
$$

i.e., $B_{a}$ does not contain any equilibria.

Theorem 2.2 [9] Consider the Hamiltonian system (6) without input, i.e. $u(t)=0$. Let $x(t)$ be the solution of the system with $x(0)=x_{\circ}$. Under Assumption 2.1, there exists $T^{*}=T^{*}(a)$ such that for all $x \in B_{a}$ and all $T \in\left(0, T^{*}\right)$, Algorithm ALG1 generates a conservative discrete-time model of the system, namely

$$
x_{k+1}=F_{T}\left(x_{k}\right)
$$

Furthermore, the discrete-time model (17) is convergent with order 2 and there is a constant $K=$ $K\left(T^{*}, a\right)$ such that, for all $x \in B_{a}$,

$$
\left\|x_{k}-x(t)\right\| \leq K T^{2},
$$

with $k T \leq t \leq(k+1) T$, for all $k \geq 0$ and $T \in\left(0, T^{*}\right)$,

Note that (14) restricts Algorithm ALG1 to apply only if $J$ is invertible, whereas singular $J$ often arises in practice. An example of this is the rigid body considered in Example 2.1. The result presented in the next section is a generalization of Algorithm ALG1, which relaxes the restriction on nonsingularity of $J$ and allows the algorithm to be used for systems with arbitrary dimension.

\section{Main Result}

\subsection{General construction}

In this section we present our main result, namely a numerical algorithm which can be seen as a generalization of Algorithm ALG1. It allows to deal with the case of singular $J$ and this automatically 
extends the applicability of the algorithm to systems of arbitrary dimension. As in Subsection 2.3, in this section we consider Hamiltonian systems with no input $(u=0)$. The formula of the new algorithm is very similar to Algorithm ALG1, with some modifications by introducing a new symmetric matrix $S$. It is written as

$$
\begin{aligned}
\frac{x_{k+1}-x_{k}}{T}= & J\left(x_{\frac{1}{2}}\right) \nabla H\left(x_{\frac{1}{2}}\right) \\
& -J\left(x_{\frac{1}{2}}\right)\left[H\left(x_{k+1}\right)-H\left(x_{k}\right)+\left\langle\nabla H\left(x_{\frac{1}{2}}\right), S\left(x_{\frac{1}{2}}\right)\left(x_{k+1}-x_{k}\right)\right\rangle\right] \frac{x_{k+1}-x_{k}}{\left\|x_{k+1}-x_{k}\right\|_{S}^{2}},
\end{aligned}
$$

where $S(\cdot):=J^{+}(\cdot) J(\cdot)$, and

$$
\left\|x_{k+1}-x_{k}\right\|_{S}^{2}:=\left(x_{k+1}-x_{k}\right)^{\top} S\left(x_{\frac{1}{2}}\right)\left(x_{k+1}-x_{k}\right) .
$$

We refer to this modified algorithm (19) as Algorithm ALG2. The Hamiltonian conservation property of Algorithm ALG2 is discussed in Proposition 3.1, and the convergence of this algorithm follows directly from Theorem 2.2 under Assumption 2.1, with the compact set $\tilde{B}_{a}:=\left\{x \in \mathbb{R}^{N}: H(x)=a\right\}$ (the system may have arbitrary dimension).

To state our results, we use the following assumptions.

A1) $\left(x_{k+1}-x_{k}\right)^{\top} S\left(x_{k+1}-x_{k}\right) \neq 0$.

A2) $\left(x_{k+1}-x_{k}\right)^{\top} S\left(x_{k+1}-x_{k}\right) \neq\left(x_{k+1}-x_{k}\right)^{\top}\left(x_{k+1}-x_{k}\right)$, for $S=J^{+} J \neq I$.

Note that we often drop the arguments of variables whenever it does not cause any confusion.

Proposition 3.1 Suppose A1) holds. Algorithm ALG2 guarantees the Hamiltonian conservation for the approximate discrete-time model.

Proof of Proposition 3.1: The proof is carried out through the following straightforward calculation. Let

$$
\begin{aligned}
\Delta_{x} & :=x_{k+1}-x_{k} \\
\Delta_{H} & :=H\left(x_{k+1}\right)-H\left(x_{k}\right) .
\end{aligned}
$$

Multiplying both sides of (19) with $T \Delta_{x}^{\top} J^{+}\left(x_{\frac{1}{2}}\right)$ yields

$$
\begin{aligned}
\Delta_{x}^{\top} J^{+} \Delta_{x} & =T \Delta_{x}^{\top} J^{+} J \nabla H\left(x_{\frac{1}{2}}\right)-T \Delta_{x}^{\top} J^{+} J \Delta_{H} \frac{\Delta_{x}}{\left\|\Delta_{x}\right\|_{S}^{2}}-T \Delta_{x}^{\top} J^{+} J\left\langle\nabla H\left(x_{\frac{1}{2}}\right), S \Delta_{x}\right\rangle \frac{\Delta_{x}}{\left\|\Delta_{x}\right\|_{S}^{2}} \\
& =T \Delta_{x}^{\top} S \nabla H\left(x_{\frac{1}{2}}\right)-T \Delta_{x}^{\top} S \Delta_{H} \frac{\Delta_{x}}{\left\|\Delta_{x}\right\|_{S}^{2}}-T \Delta_{x}^{\top} S \frac{\Delta_{x}}{\left\|\Delta_{x}\right\|_{S}^{2}}\left\langle\nabla H\left(x_{\frac{1}{2}}\right), S \Delta_{x}\right\rangle .
\end{aligned}
$$

It can be easily seen that the first and the third terms of (20) eliminate each other, and hence we are left with

$$
\begin{aligned}
\Delta_{x}^{\top} J^{+} \Delta_{x} & =-T \Delta_{x}^{\top} S \Delta_{H} \frac{\Delta_{x}}{\left\|\Delta_{x}\right\|_{S}^{2}} \\
& =-T \Delta_{H} \frac{\Delta_{x}^{\top} S \Delta_{x}}{\left\|\Delta_{x}\right\|_{S}^{2}} \\
& =-T \Delta_{H} .
\end{aligned}
$$


Since $J^{+}$is a skew-symmetric matrix, the left-hand side of (20) is zero. Hence, this implies

$$
H\left(x_{k+1}\right)=H\left(x_{k}\right)
$$

which completes the proof of Hamiltonian conservation.

Remark 3.1 Algorithm ALG2 can be further generalized not only to obtain Hamiltonian conservation, but also to include other conserved quantities, e.g. Casimir functions, by replacing the term $H\left(x_{k+1}\right)-$ $H\left(x_{k}\right)$ with

$$
\sqrt{\sum_{i=1}^{j}\left(\mathcal{H}_{i}\left(x_{k+1}\right)-\mathcal{H}_{i}\left(x_{k}\right)\right)^{2}},
$$

where $\mathcal{H}_{i}, i=1, \cdots, j$, denote the conserved quantities.

\subsection{Preservation of the losslessness property}

In Subsection 3.1 it has been shown that Algorithm ALG2 guarantees Hamiltonian conservation for systems without input. When considering port-controlled Hamiltonian systems (6), we are then dealing with systems with (control) inputs. In [10], port-controlled Hamiltonian systems are seen as passive systems. As shown by (12), considering the Hamiltonian $H$ as the storage function of the system, a continuous-time conservative port-controlled Hamiltonian system is lossless [10, 12]. Now, if we use Algorithm ALG2 to discretize the port-controlled Hamiltonian system (6), taking into account the input $u$, we will show that the discretization yields a preservation of a generalized form of the losslessness property. We use the notation $x_{k+1}^{u}$ to denote the state $x_{k+1}$ with the control input $u \neq 0$, and define Algorithm ALG2 for the port-controlled Hamiltonian system (6) with $u \neq 0$ as follows:

$$
\frac{\Delta_{x}^{u}}{T}=J\left(x_{\frac{1}{2}}\right) \nabla H\left(x_{\frac{1}{2}}\right)+g\left(x_{\frac{1}{2}}\right) u-J\left(x_{\frac{1}{2}}\right)\left[\Delta_{H}+\left\langle\nabla H\left(x_{\frac{1}{2}}\right), S\left(x_{\frac{1}{2}}\right)\left(x_{k+1}-x_{k}\right)\right\rangle\right] \frac{x_{k+1}-x_{k}}{\left\|x_{k+1}-x_{k}\right\|_{S}^{2}} .
$$

Note that we have chosen the position of the term $g\left(x_{\frac{1}{2}}\right) u$ in (23) in such a way to avoid the problem of multiplying $u$ with the singular structure matrix $J(\cdot)$ as if we included the term containing $u$ in the bracket with $\Delta_{H}$. By this definition, we have

$$
\Delta_{x}^{u}=x_{k+1}^{u}-x_{k}=x_{k+1}-x_{k}+T g\left(x_{\frac{1}{2}}\right) u .
$$

Applying this to compute the Hamiltonian difference for the system (6) yields

$$
\begin{aligned}
\Delta_{H}^{u} & =H\left(x_{k+1}^{u}\right)-H\left(x_{k}\right) \\
& =H\left(x_{k+1}^{u}\right)-H\left(x_{k+1}\right)+\underbrace{H\left(x_{k+1}\right)-H\left(x_{k}\right)}_{0} \\
& =\nabla H^{\top}(\tilde{x})\left[x_{k+1}^{u}-x_{k+1}\right] \\
& =T \nabla H^{\top}(\tilde{x}) g\left(x_{\frac{1}{2}}\right) u,
\end{aligned}
$$

where

$$
\tilde{x}:=x_{k+1}+\theta T g\left(x_{\frac{1}{2}}\right) u, \text { for } \theta \in(0,1) .
$$


From the continuity of $\nabla H(\cdot)$ and $g(\cdot)$, we can always write

$$
\nabla H(\tilde{x})=\nabla H\left(x_{k}\right)+T \phi_{H}\left(x_{k}, u\right) \text { and } g\left(x_{\frac{1}{2}}\right)=g\left(x_{k}\right)+T \phi_{g}\left(x_{k}, u\right)
$$

with $\phi_{H}(0,0)=0, \phi_{g}(0,0)=0$ and both functions are continuous. Therefore,

$$
\begin{aligned}
\Delta_{H}^{u} & =T \nabla H^{\top}(\tilde{x}) g\left(x_{\frac{1}{2}}\right) u \\
& =\left(\nabla H\left(x_{k}\right)+T \phi_{H}\right)^{T}\left(g\left(x_{k}\right)+T \phi_{g}\right) u \\
& =\left[T \nabla H^{\top}(x) g(x)+O\left(T^{2}\right)\right] u=\left[T y^{\top}+O\left(T^{2}\right)\right] u,
\end{aligned}
$$

which shows that the losslessness property is preserved with an offset of order $T^{2}$. Moreover, the Hamiltonian is still conserved when $u=0$.

Remark 3.2 Note that Algorithm ALG2 does not compute the exact discrete-time model of the system, but it computes an approximate model that contains some discrepancies from the exact model (see Theorem 2.2). Due to these discrepancies, it is expected that the 'strict' preservation of losslessness does not hold and the best we can get is the preservation of losslessness with an offset of order $T^{2}$ (when $u \neq 0$ ). This result is comparable with the preservation of dissipativity property under sampling presented in $[3]$.

\section{Application to systems with quadratic Hamiltonian}

Motivated by the application of Algorithm ALG1 to linear systems [9], in this subsection, we focus on applying Algorithm ALG2 to a subclass of port-controlled Hamiltonian systems with quadratic Hamiltonian, i.e.

$$
H(x)=\frac{1}{2} x^{\top} A x \quad \text { and } \quad \nabla H(x)=A x
$$

with $A$ a positive definite symmetric matrix, and the matrix $J(x)$ a linear function of $x$, which satisfies

$$
J(a x)=a J(x), \quad \forall a \in \mathbb{R} .
$$

Corollary 4.1 Suppose A1) and A2) hold. Consider the port-controlled Hamiltonian systems (6), with $u=0$, structure matrix $J(x)$ satisfying (29), and Hamiltonian $H(x)$ satisfying (28). Then the following holds.

C1. Algorithm ALG2 guarantees Hamiltonian conservation for the approximate discrete-time model of the system.

C2. Algorithm ALG2 reduces to an implicit midpoint algorithm for the system.

Proof of Corollary 4.1: The proof of C1 is a direct consequence of Proposition 3.1, and here we concentrate on the proof of C2. From C1, we have that the Hamiltonian is conserved. Therefore,

$$
\Delta_{H}=H\left(x_{k+1}\right)-H\left(x_{k}\right)=0,
$$


and Algorithm ALG2 can be written as

$$
\frac{\Delta_{x}}{T}=J\left(x_{\frac{1}{2}}\right) \nabla H\left(x_{\frac{1}{2}}\right)-J\left(x_{\frac{1}{2}}\right)\left\langle\nabla H\left(x_{\frac{1}{2}}\right), S \Delta_{x}\right\rangle \frac{\Delta_{x}}{\left\|\Delta_{x}^{2}\right\|_{S}} .
$$

We claim the following:

Claim. If $J(x)$ and $H(x)$ are as specified in Corollary 4.1 then $\left\langle\nabla H\left(x_{\frac{1}{2}}\right), S\left(x_{\frac{1}{2}}\right) \Delta_{x}\right\rangle=0$.

Proof of Claim. To prove the claim, we consider two cases.

Case 1: $S=J^{+} J \neq I$

Using properties (4)-(5) and by the fact that Algorithm ALG2 is Hamiltonian conserving, we have

$$
\begin{aligned}
\left\langle\nabla H\left(x_{\frac{1}{2}}\right), S \Delta_{x}\right\rangle=\Delta_{x}^{\top} S \nabla H\left(x_{\frac{1}{2}}\right) & \\
= & T\left(J\left(x_{\frac{1}{2}}\right) \nabla H\left(x_{\frac{1}{2}}\right)\right)^{\top} S \nabla H\left(x_{\frac{1}{2}}\right)-T\left(J\left(x_{\frac{1}{2}}\right) \frac{\left\langle\nabla H\left(x_{\frac{1}{2}}\right), S \Delta_{x}\right\rangle}{\left\|\Delta_{x}\right\|_{S}^{2}} \Delta_{x}\right)^{\top} S \nabla H\left(x_{\frac{1}{2}}\right) \\
& =-T \frac{\left\langle\nabla H\left(x_{\frac{1}{2}}\right), S \Delta_{x}\right\rangle}{\left\|\Delta_{x}\right\|_{S}^{2}} \Delta_{x}^{\top}\left(J\left(x_{\frac{1}{2}}\right)\right)^{\top} \nabla H\left(x_{\frac{1}{2}}\right) \\
& =T \frac{\left\langle\nabla H\left(x_{\frac{1}{2}}\right), S \Delta_{x}\right\rangle}{\left\|\Delta_{x}\right\|_{S}^{2}} \Delta_{x}^{\top} J\left(x_{\frac{1}{2}}\right) \nabla H\left(x_{\frac{1}{2}}\right) .
\end{aligned}
$$

From Algorithm ALG2, we have

$$
\begin{aligned}
T \Delta_{x}^{\top} J\left(x_{\frac{1}{2}}\right) \nabla H\left(x_{\frac{1}{2}}\right) & =\Delta_{x}^{\top}\left(\Delta_{x}+T J\left(x_{\frac{1}{2}}\right) \frac{\left\langle\nabla H\left(x_{\frac{1}{2}}\right), S \Delta_{x}\right\rangle}{\left\|\Delta_{x}\right\|_{S}^{2}} \Delta_{x}\right) \\
& =\Delta_{x}^{\top} \Delta_{x}+T \frac{\left\langle\nabla H\left(x_{\frac{1}{2}}\right), S \Delta_{x}\right\rangle}{\left\|\Delta_{x}\right\|_{S}^{2}} \Delta_{x}^{\top} J\left(x_{\frac{1}{2}}\right) \Delta_{x}=\Delta_{x}^{\top} \Delta_{x} .
\end{aligned}
$$

Hence, we obtain

$$
\left\langle\nabla H\left(x_{\frac{1}{2}}\right), S \Delta_{x}\right\rangle=\left\langle\nabla H\left(x_{\frac{1}{2}}\right), S \Delta_{x}\right\rangle \frac{\Delta_{x}^{\top} \Delta_{x}}{\left\|\Delta_{x}\right\|_{S}^{2}}
$$

By the assumption A2), $\frac{\Delta_{x}^{\top} \Delta_{x}}{\left\|\Delta_{x}\right\|_{S}^{2}} \neq 1$, which implies that $\left\langle\nabla H\left(x_{\frac{1}{2}}\right), S \Delta_{x}\right\rangle=0$.

\section{Case 2: $S=J^{+} J=I$}

This case follows [9] similarly. Substitute $S=I$ and from the fact that $\Delta_{H}=\frac{1}{2} x_{k+1}^{\top} A x_{k+1}-\frac{1}{2} x_{k}^{\top} A x_{k}=$ 0 we obtain

$$
\begin{aligned}
\left\langle\nabla H\left(x_{\frac{1}{2}}\right), \Delta_{x}\right\rangle & =\Delta_{x}^{\top} \nabla H\left(x_{\frac{1}{2}}\right) \\
& =\left(x_{k+1}-x_{k}\right)^{\top} A\left(x_{k+1}+x_{k}\right) \\
& =\left(x_{k+1}\right)^{\top} A x_{k+1}-\left(x_{k}\right)^{\top} A x_{k}+\left(x_{k+1}\right)^{\top} A x_{k}-\left(x_{k}\right)^{\top} A x_{k+1}=0,
\end{aligned}
$$

which proves the claim.

Therefore, Algorithm ALG2 reduces to

$$
\begin{aligned}
\frac{\Delta_{x}}{T} & =J\left(x_{\frac{1}{2}}\right) \nabla H\left(x_{\frac{1}{2}}\right) \\
& =J\left(\frac{1}{2} x_{k+1}+\frac{1}{2} x_{k}\right) A\left(\frac{1}{2} x_{k+1}+\frac{1}{2} x_{k}\right) \\
& =\frac{1}{4} J\left(x_{k+1}+x_{k}\right) A\left(x_{k+1}+x_{k}\right),
\end{aligned}
$$

which turns out to be the implicit midpoint algorithm, and this completes the proof. 


\section{Stabilization of port-controlled Hamiltonian systems}

As mentioned earlier, in order to carry out a direct discrete-time controller design we require a 'good' and 'well behaved' discrete-time model of the plant. The result we have presented in the previous section provides the tool to construct such model. In this section we use this tool and consider the stabilization problem for port-controlled Hamiltonian systems (6). The design is aiming to construct a controller to be implemented as a piecewise constant signal $u_{t}=u_{k T}=: u_{k}, \forall t \in[k T,(k+1) T), k \in \mathbb{N}$.

Proposition 5.1 Consider port-controlled Hamiltonian system (6). Assume that the nominal system without control is detectable and the equilibrium point to be stabilized is the origin. A discrete-time approximate model of the system obtained by Algorithm ALG2 is semiglobally stabilized by the discretetime feedback

$$
u=u_{k}=-c g\left(x_{\frac{1}{2}}\right)^{\top} \nabla H\left(x_{k+1}\right),
$$

with $c>0$.

Proof of Proposition 5.1: Let $x_{k+1}^{u}$ be obtained using Algorithm ALG2, taking into account the control $u$ as in (23). Following the same computation as in Section 3, we arrived at (25) with $\tilde{x}$ given by (26). From the continuity of $\nabla H(\cdot)$, we can always write

$$
\nabla H(\tilde{x})=\nabla H\left(x_{k+1}\right)+T \psi_{u}\left(x_{k}, u\right)
$$

with $\psi_{u}(\cdot, 0)=0$ and continuous. Therefore we can write

$$
\psi_{u}\left(x_{k}, u\right):=\tilde{\psi}_{u}\left(x_{k}, u\right) u
$$

Hence, applying the controller (36) to (25) gives

$$
\begin{aligned}
\Delta_{H}^{u} & =T \nabla H^{\top}(\tilde{x}) g\left(x_{\frac{1}{2}}\right) u_{k} \\
& =T\left(\left(\nabla H\left(x_{k+1}\right)+T \tilde{\psi}_{u}\left(x_{k}, u_{k}\right) u_{k}\right)^{\top} g\left(x_{\frac{1}{2}}\right)\right) u_{k} \\
& \leq-c T\left(\nabla H\left(x_{k+1}\right)^{\top} g\left(x_{\frac{1}{2}}\right)\right)\left(\nabla H^{\top}\left(x_{k+1}\right) g\left(x_{\frac{1}{2}}\right)\right)^{\top}+T^{2}\left(u_{k}\right)^{\top} \underbrace{\tilde{\psi}_{u}^{\top}\left(x_{k}, u_{k}\right) g\left(x_{\frac{1}{2}}\right)}_{\hat{\psi}_{u}} u_{k} \\
& \leq-\frac{T}{c}\left(u_{k}\right)^{\top} u_{k}+T^{2}\left(u_{k}\right)^{\top} \hat{\psi}_{u} u_{k} \\
& =-T\left(u_{k}\right)^{\top}\left(\frac{1}{c}-T \hat{\psi}_{u}\right) u_{k} .
\end{aligned}
$$

For any fix $c>0$, we can always pick $T>0$ such that $\left(\frac{1}{c}-T \hat{\psi}_{u}\right) \geq 0$. Since $\hat{\psi}_{u}$ is zero when $x_{k}$ is zero, with the detectability property of the nominal system, the closed-loop system is semiglobally practically asymptotically stable. The semiglobal property comes from the fact that $T$ is a function of the set in which the approximate model is defined.

For port-controlled Hamiltonian systems with quadratic Hamiltonian, we can state the following corollary. 
Corollary 5.1 Consider system (6) with the structure matrix $J(x)$ satisfying (29) and the Hamiltonian $H(x)$ satisfying (28), with $A>0$. Assume that the nominal system without control is detectable and the equilibrium point to be stabilized is the origin. A discrete-time approximate model of the system obtained by Algorithm ALG2 is semiglobally practically stabilized by the discrete-time feedback $u=$ $u_{k}=-c g\left(x_{\frac{1}{2}}\right)^{\top} A x_{k+1}$, where $c>0$.

Proof of Corollary 5.1: Following exactly the same steps as the proof of Proposition 5.1, and then substituting (28) into (25) yields $\Delta_{H}^{u}=T \tilde{x}^{\top} A g\left(x_{\frac{1}{2}}\right) u$. Applying $u_{k}=-c g\left(x_{\frac{1}{2}}\right)^{\top} A x_{k+1}$ gives

$$
\begin{aligned}
\Delta_{H}^{u} & =T \tilde{x}^{\top} A g\left(x_{\frac{1}{2}}\right) u_{k} \\
& =T\left(x_{k+1}+\theta T g\left(x_{\frac{1}{2}}\right) u_{k}\right)^{\top} A g\left(x_{\frac{1}{2}}\right) u_{k} \\
& =-c T x_{k+1}^{\top} A g\left(x_{\frac{1}{2}}\right)\left(x_{k+1}^{\top} A g\left(x_{\frac{1}{2}}\right)\right)^{\top}+\theta T^{2}\left(g\left(x_{\frac{1}{2}}\right) u_{k}\right)^{\top} A g\left(x_{\frac{1}{2}}\right) u_{k} \\
& \leq-c T x_{k+1}^{\top} A g\left(x_{\frac{1}{2}}\right)\left(x_{k+1}^{\top} A g\left(x_{\frac{1}{2}}\right)\right)^{\top}+T^{2}\left(u_{k}\right)^{\top} \underbrace{\left(g\left(x_{\frac{1}{2}}\right)\right)^{\top} \theta A g\left(x_{\frac{1}{2}}\right)}_{\hat{\psi}_{u}} u_{k} .
\end{aligned}
$$

Continuing the calculation similarly to (38), the same conclusion as in Proposition 5.1 is reached.

Note that Proposition 5.1 shows the stabilization of the approximate discrete-time model, whereas in sampled-data control design we are interested in the stabilization of the original continuous-time plant with the designed discrete-time controller. In [6, 4], a design framework that relates the stability of the approximate discrete-time model, the exact model and the sampled-data system has been provided and all conditions required for the framework $[6,4]$ are satisfied in our case. Therefore we can use the results of $[6,4]$ to directly conclude stability of the sampled-data system.

Remark 5.1 If $\tilde{x}=x_{k}+T f\left(x_{k}\right)$ and $g(x)=g$ is a constant vector, we obtain that the discrete-time controller $u_{k}$ takes form

$$
u_{k}=u_{c}+T g^{\top} A f\left(x_{k}\right),
$$

where $u_{c}$ is the sampled version of a continuous-time stabilizing controller of the system. Some studies show that compared with the control $u_{k}^{c}$, the extra term $T g^{\top} A f\left(x_{k}\right)$ in $u_{k}$ helps improving the closedloop response (see $[2,11])$.

Example 4.1 Consider the rigid body dynamics given in Example 2.1, with the principal moments of inertia $I_{1}=3, I_{2}=2, I_{3}=1$, and the Hamiltonian

$$
H=\frac{1}{2}\left(3 \alpha^{2}+2 \beta^{2}+\gamma^{2}\right)
$$

The dynamics of the system can be written as

$$
\begin{aligned}
\dot{\alpha} & =\frac{1}{3} \beta \gamma+\frac{1}{3} u \\
\dot{\beta} & =-\alpha \gamma+\frac{1}{2} u \\
\dot{\gamma} & =\alpha \beta+u .
\end{aligned}
$$


It was shown in Example 2.1 that the system with $u=0$ is conservative, and

$$
u_{c}=-c g^{\top} \nabla H(x)=-c(\alpha+\beta+\gamma), \quad c>0,
$$

is a stabilizing controller for the system (42) (see also [7]). Since (42) satisfies the requirement for Corollary 5.1, we can apply the implicit midpoint algorithm and obtain the discrete-time model of the system as

$$
\begin{aligned}
\alpha_{k+1} & =\alpha_{k}+\frac{1}{12} T \Sigma_{\beta} \Sigma_{\gamma}+\frac{1}{3} T u_{k} \\
\beta_{k+1} & =\beta_{k}-\frac{1}{4} T \Sigma_{\gamma} \Sigma_{\alpha}+\frac{1}{2} T u_{k} \\
\gamma_{k+1} & =\gamma_{k}+\frac{1}{4} T \Sigma_{\alpha} \Sigma_{\beta}+T u_{k}
\end{aligned}
$$

with $\Sigma_{\alpha}:=\alpha_{k}+\alpha_{k+1}, \Sigma_{\beta}:=\beta_{k}+\beta_{k+1}$ and $\Sigma_{\gamma}:=\gamma_{k}+\gamma_{k+1}$. Applying Corollary 5.1, the semiglobally asymptotically stabilizing controller for the discrete-time model (44) is

$$
u_{k}=-c\left(\alpha+\beta+\gamma+T\left(\frac{1}{12} \Sigma_{\beta} \Sigma_{\gamma}-\frac{1}{4} \Sigma_{\gamma} \Sigma_{\alpha}+\frac{1}{4} \Sigma_{\alpha} \Sigma_{\beta}\right)\right) .
$$

Note that since the model (44) is obtained using an implicit algorithm, the controller (45) is a recursive function of $x_{k+1}$. To limit the recursion, the truncation up to $T$-terms is implemented, i.e.

$$
u_{k}=-c\left(\alpha+\beta+\gamma+4 T\left(\frac{1}{12} \beta \gamma-\frac{1}{4} \gamma \alpha+\frac{1}{4} \alpha \beta\right)\right),
$$

which yields the Hamiltonian difference

$$
\Delta_{H}^{u}=-c T\left(\alpha+\beta+\gamma+4 T\left(\frac{1}{12} \beta \gamma-\frac{1}{4} \gamma \alpha+\frac{1}{4} \alpha \beta\right)\right)^{2}+O\left(T^{2}\right) .
$$

Hence the controller $u_{k}$ stabilizes the discrete-time model in a semiglobal practical sense and it stabilizes the sampled data systems.

The response of the closed-loop system when applying the midpoint based controller (46) to the continuous-time plant (42), compared to those when applying the emulation controller that is obtained by sampling the continuous-time controller (43) letting $c=2$, are shown in Figure 1 and the corresponding Hamiltonians are shown in Figure 2. In the simulation we have used $T=0.1 s$, and $x_{\circ}=(252525)^{\top}$.

It is shown that the midpoint based controller outperforms the emulation controller, in terms of transient response. Figure 1 shows that the midpoint based controller reduces the oscillation of the states. Further simulations show that this phenomena is consistent for large initial states, while both controllers give very similar responses for small initial states.

\section{Summary}

We have presented results on discretization of Hamiltonian systems. A numerical algorithm that guarantees Hamiltonian conservation for systems with arbitrary dimension and possibly singular structure matrix has been proposed. The algorithm is also shown to preserve the losslessness of port-controlled 

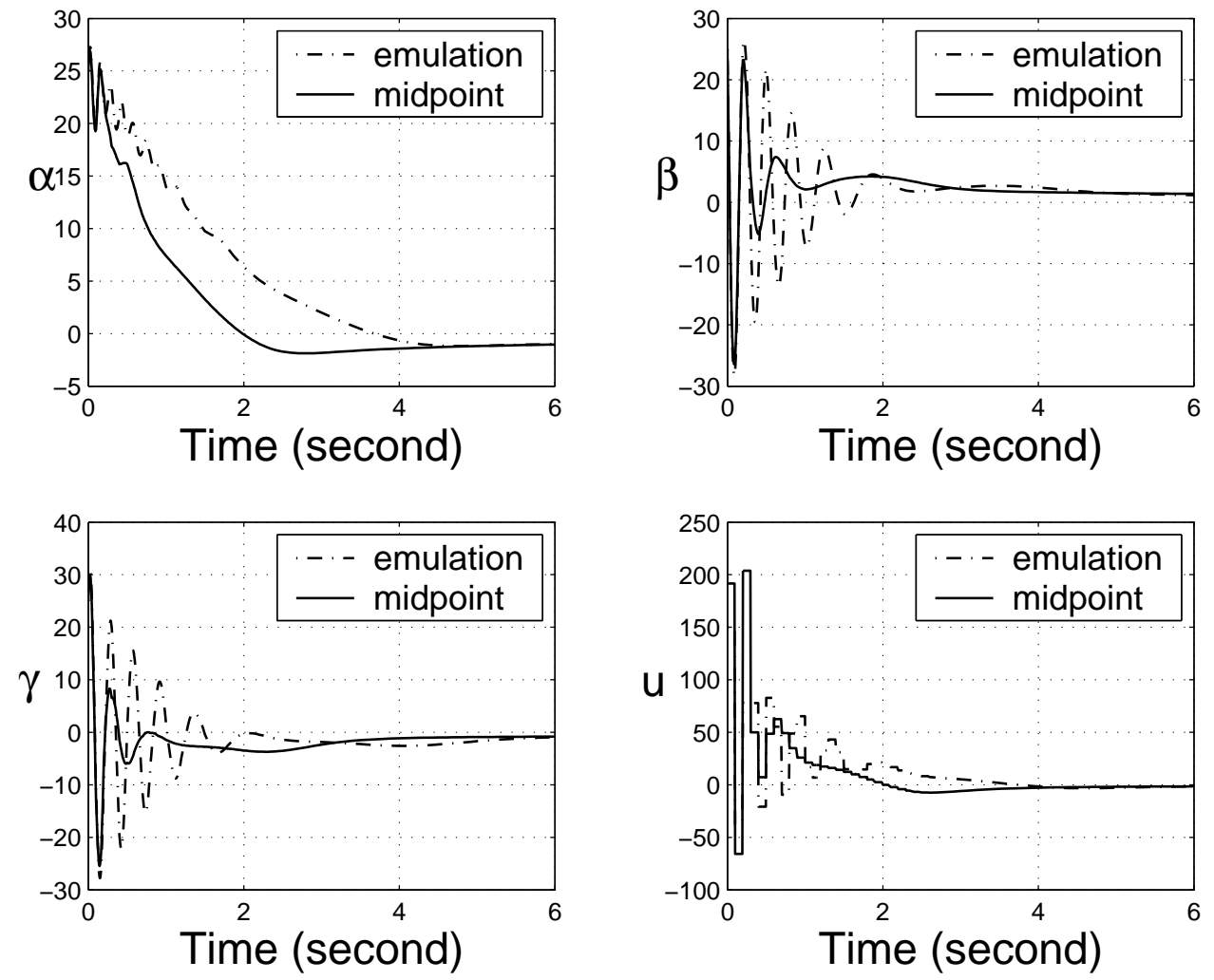

Figure 1: Response with midpoint based controller $u_{k}$ and emulation controller $u_{k}^{c}$.

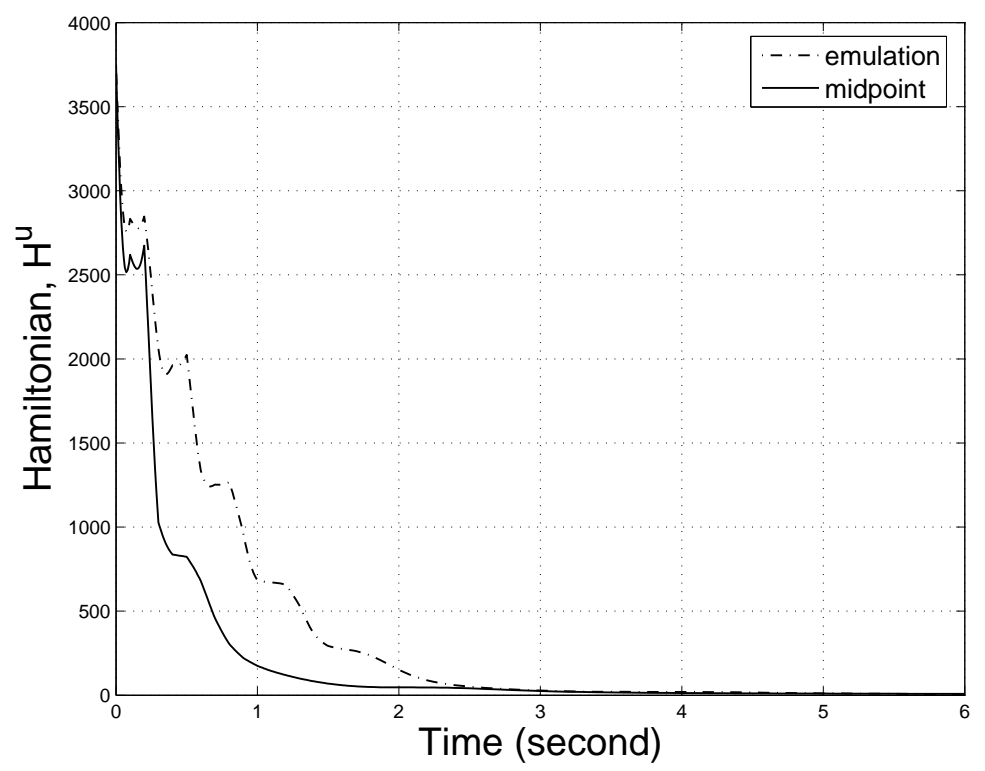

Figure 2: Hamiltonian with midpoint based controller $u_{k}$ and emulation controller $u_{k}^{c}$. 
Hamiltonian systems. We have applied the algorithm to port-controlled Hamiltonian systems with quadratic Hamiltonian, and finally, we have shown the usefulness of the algorithm for discrete-time controller design based on Hamiltonian conserving approach. This result gives a strong motivation for further investigation on sampled-data stabilization design for Hamiltonian conservative systems.

\section{References}

[1] O. Gonzalez. Time integration and discrete Hamiltonian systems. Jour. Nonlinear Science, 6:449$467,1996$.

[2] D. S. Laila and D. Nešić. Changing supply rates for input-output to state stable discrete-time nonlinear systems with applications. Automatica, 39:821-835, 2003.

[3] D. S. Laila, D. Nešić, and A. R. Teel. Open and closed loop dissipation inequalities under sampling and controller emulation. European Journal of Control, 8:109-125, 2002.

[4] D. Nešić and A. R. Teel. A framework for stabilization of nonlinear sampled-data systems based on their approximate discrete-time models. IEEE Trans. Auto. Contr., 49:1103-1122, 2004.

[5] D. Nešić, A. R. Teel, and P. Kokotović. Sufficient conditions for stabilization of sampled-data nonlinear systems via discrete-time approximations. Syst. Contr. Lett., 38:259-270, 1999.

[6] D. Nešić, A. R. Teel, and E. Sontag. Formulas relating $\mathcal{K} \mathcal{L}$ stability estimates of discrete-time and sampled-data nonlinear systems. Syst. Contr. Lett., 38:49-60, 1999.

[7] R. Outbib and G. Sallet. Stabilizability of the angular velocity of a rigid body revisited. Syst. Cont. Lett., 18:93-98, 1992.

[8] J. M. Sanz-Serna and M. P. Calvo. Numerical Hamiltonian Problems. Chapman \& Hall, 1994.

[9] A. M. Stuart and A. R. Humphries. Dynamical Systems and Numerical Analysis. Cambridge Univ. Press, New York, 1996.

[10] A. van der Schaft. $L_{2}$-Gain and Passivity Techniques in Nonlinear Control. Springer, 2000.

[11] G. D. Warshaw and H. M. Schwartz. Sampled-data robot adaptive control with stabilizing compensation. The International Journal of Robotic Research, 15:78-91, 1996.

[12] J. C. Willems. Dissipative dynamical systems part I, part II. Archive for Rational Mechanics and Analysis, 45:325-393, 1972. 